Rev. Biol. Neotrop. 3(2): 157-162. 2006

\title{
valiação da atividade Mutagênica e Genotóxica de Ginkgo BILOBA L. PELO TESTE DO MICRONÚCLEO EM CAMUNDONGOS
}

\author{
Kaio Ramos Leite \\ Laryssa Silva Andrade \\ Juliana Stival Sena \\ Juliana B. Vilar \\ Lee Chen Chen \\ Laboratório de Radiobiologia e Mutagênese, Departamento de Biologia Geral, ICB, Universi- \\ dade Federal de Goiás, Campus-II, 74001-970, Goiânia, Goiás, Brasil; email: chenlee@icb. \\ ufg.br
}

\begin{abstract}
Resumo: Ginkgo biloba L. é uma planta medicinal amplamente utilizada pela população mundial para o tratamento de insuficiência cerebral, doenças vasculares e outras enfermidades. No presente trabalho foi avaliada a atividade clastogênica e/ou aneugênica do extrato das folhas de Ginkgo biloba L. (EGb 761) pelo teste de micronúcleo em eritrócitos policromáticos da medula óssea de camundongos. Grupos de cinco animais foram tratados com extrato EGb 761 por via gavage com doses de $50 \mathrm{mg} / \mathrm{kg}, 100 \mathrm{mg} / \mathrm{kg}$ e $200 \mathrm{mg} / \mathrm{kg}$. Para todas as doses utilizadas, a freqüência de eritrócitos policromáticos micronucleados (EPCMN) foi avaliada no tempo de 24 horas pós-tratamento. A preparação citológica foi realizada de acordo com a metodologia de Heddle. A citotoxidade foi avaliada pela relação de eritrócitos policromáticos e normocromáticos (EPC/ENC). Os resultados mostraram que o EGb 761 não provocou aumento significativo $(p>0,05)$ na freqüência de EPCMN em relação ao grupo controle negativo. Não foi observada citotoxicidade para todas as concentrações utilizadas $(P>0,01)$ em relação ao grupo controle negativo. Dessa maneira, pode-se sugerir que EGb 761 não apresentou atividade mutagênica e/ou citotóxica nas condições experimentais executadas.
\end{abstract}

Palavras-chave: camundongos, citotoxicidade, extrato Ginkgo biloba, mutagenicidade, teste do micronúcleo.

Aвstract: Ginkgo biloba L. is a medicinal plant widely used worldwide for the treatment of cerebral insufficiency, vascular diseases and various other illnesses. In the present study, the clastogenic and/or aneugenic activities of G. biloba L. leaf extract (EGB 761) were evaluated using mouse bone marrow micronucleus assay in polycromatic erythrocytes. Groups of five animals were treated with EGB 761 by gavage administration in doses of $50 \mathrm{mg} / \mathrm{kg}, 100 \mathrm{mg} / \mathrm{kg}$, and $200 \mathrm{mg} / \mathrm{kg}$. For all the doses, the micronucleated polycromatic erythrocytes frequency (MNPCE) was evaluated after 24 hours of treatment. The cytological preparation was carried out according to Heddle methodology. The cytotoxicity was evaluated by the relationship between the polycromatic and normocromatic frequency (EPC/ENC). The results indicated that EGb 761 did not provoke a significant increase of MNPCE $(P>0.05)$ compared to the negative control group. Cytotoxicity was not observed in all concentrations utilized $(P>0.01)$ compared to the negative control group. Thus, it can be suggested that EGb 761 did not present mutagenic nor cytotoxic effects under these experimental conditions.

Key words: mice, cytotoxicity, extract Ginkgo biloba, mutagenicity, micronucleus test.

\section{INTRODUÇÃo}

$\Lambda_{\text {s plantas sempre foram empregadas }}$ como fonte comum de medicamentos. Atualmente, estima-se que mais de $80 \%$ da população mundial utiliza plantas como fonte primária de diversos agentes medicinais (Cordell, 1995). Entretanto, os compostos derivados de plantas 
podem conter constituintes que exercem efeitos colaterais indesejáveis (Shaw et al., 1997). Assim, os fitoterápicos devem ser avaliados sob diversos aspectos, tais como eficácia, qualidade e segurança (Couzinier \& Mamatas, 1986; Saller et al., 1998; De Smet, 1995).

Ginkgo biloba L. (Ginkgoaceae) é uma árvore de origem chinesa que tem sido extensivamente cultivada na Europa, Austrália, Japão, Coréia e Estados Unidos (Kunitomo et al., 2002). O uso medicinal dessa planta pode ser remontado há 5000 anos, na tradicional medicina chinesa. $\mathrm{O}$ extrato padronizado derivado dessa planta, denominado Extrato de Ginkgo biloba (EGb 761), é atualmente o fitoterápico mais receitado na Inglaterra e França para tratamento de má circulação cerebral e doenças vasculares, embora muitos estudos sugiram que pode ser capaz de atuar também na cognição, memória, ansiedade e regulação gênica (Auclair et al., 2004; Chen et al., 2005).

Os principais constituintes ativos do extrato medicinal de folhas de G. biloba incluem 22-27\% de flavonóides glicosídicos, 5-7\% de terpenos lactonas e menos que $5 \mathrm{mg} / \mathrm{g}$ de ácidos ginkgólicos (DeFeudis, 1998).

Vários estudos realizados anteriormente mostraram que as folhas de G. biloba desempenham papéis importantes no melhoramento da circulação, particularmente a circulação cerebral. (Kleijnen \& Knipschild, 1992; Oken et al., 1998; Wadsworth \& Koop, 2001). Também desempenham funções importantes na circulação vascular, memória, função cognitiva, ansiedade e regulação gênica (Cohen-Salmon et al., 1997; Elovic \& Zafonte, 2001; Thiagaraja et al., 2002; DeFeudis, 2003; Rioufol et al., 2003). Muitos dos efeitos farmacológicos de G. biloba são atribuídos aos flavonóides e terpenos presentes em seus extratos (Porsolt et al., 1990; Rapin et al., 1994; Amri et al., 1996; Bastianetto \& Quirion, 2002; Zhao \& Li, 2002).

Entretanto, o EGb 761 pode conter constituintes que apresentam ações citotóxicas, genotóxicas, alergênicas e imunotóxicas (Jaggy \& Koch, 1997; Siegers, 1999; Westen-dorf \& Regan, 2000; Koch et al., 2000; Ahlemeyer et al., 2001). Assim, é de grande interesse que se consiga identificar compostos com propriedades farmacológicas e que não apresentem atividade genotóxica.

Tendo em vista a ampla utilização do $G$. biloba como fitoterápico pela população mundial, torna-se importante avaliar possíveis efeitos adversos que o extrato possa vir causar à saúde humana. No presente trabalho foi avaliado o potencial mutagênico e citotóxico do extrato de folhas de G. biloba (EGb 761) pelo teste do micronúcleo em eritrócitos de medula óssea de camundongos.

O teste do micronúcleo em medula óssea de roedores in vivo visa detectar e quantificar a ação mutagênica e/ou anti-mutagênica de agentes indutores, sendo amplamente aceito pelas agências internacionais e instituições governamentais como parte de uma bateria de testes recomendada para se estabelecer a avaliação e o registro de novos produtos químicos e farmacêuticos que entram anualmente no mercado mundial (Choy, 2001). Esse ensaio apresenta algumas vantagens em relação aos outros, entre as quais podem ser citadas sensibilidade, custo e confiabilidade. Esse teste pode ser executado praticamente em qualquer população de células que esteja em constante divisão, sendo a medula óssea de mamíferos uma das regiões mais adequadas, visto que suas células levam de 22 a 24 horas para completar um ciclo de divisão (Heddle, 1973).

\section{Material e Métodos}

\section{Camundongos}

No presente estudo foram utilizados 25 camundongos machos, saudáveis, da espécie Mus musculus out bred, linhagem Swiss Webster, procedentes do Biotério Central da Universidade Federal de Goiás, Goiânia, pesando entre 30 e $40 \mathrm{~g}$, com idade de 8 a 12 semanas. Os animais foram trazidos 5 dias antes da realização do experimento para o laboratório, onde foram mantidos à temperatura ambiente, que ficou em torno de $25 \pm 2{ }^{\circ} \mathrm{C}$. Durante esse período, os camundongos foram mantidos em gaiolas de plástico

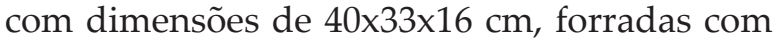
serragem de madeira trocada diariamente. Os animais foram alimentados com ração comercial e água oferecidas "ad libitum". Em cada gaiola foram colocados no máximo cinco animais e pelo menos após cinco dias de permanência no local é que foram iniciados os experimentos.

\section{Extrato de Ginkgo biloba (EGb 761)}

O extrato de G. biloba EGb 761 (lot. número: GB001128) foi obtido na Índia por intermédio da Faculdade de Farmácia da Universidade 
Federal de Goiás.

\section{Drogas e Reagentes}

Neste estudo foram utilizados os seguintes reagentes e drogas : Mitomicina $C$ (MMC) - $\mathrm{C}_{15} \mathrm{H}_{18} \mathrm{~N}_{4} \mathrm{O}_{5}$ (Bristol-Myers Squibb), giemsa (Doles), metanol P.A. - $\mathrm{CH}_{4} \mathrm{O}$ (Ecibra), soro bovino fetal inativado/estéril (Nutricell), fosfato de sódio dibásico - $\mathrm{Na}_{2} \mathrm{HPO}_{4} 7 \mathrm{H}_{2} \mathrm{O} \mathrm{P}$. A. (Vetec), fosfato de sódio monobásico P. A. - $\mathrm{NaH}_{2} \mathrm{PO}_{4} \mathrm{H}_{2} \mathrm{O}$ (Vetec).

\section{Procedimento Experimental}

Grupos de cinco animais foram tratados, via gavage, com doses de $50 \mathrm{mg} / \mathrm{kg}, 100 \mathrm{mg} / \mathrm{kg}$ e $200 \mathrm{mg} / \mathrm{kg}$ de EGb 761. No controle negativo foi utilizada $\mathrm{H}_{2} \mathrm{O}$ destilada esterilizada e no controle positivo, $4 \mathrm{mg} / \mathrm{kg}$ de MMC. Decorridas 24 horas o tratamento, os animais foram sacrificados por deslocamento cervical e tiveram ambos os fêmures retirados. As epífises dos fêmures foram então cortadas e a medula óssea aspirada com $1 \mathrm{~mL}$ de soro bovino fetal (SBF). Após completa homogeneização da medula no soro, esta foi centrifugada a $300 x y$ por $5 \mathrm{~min}$. O sobrenadante foi parcialmente descartado e o precipitado de células foi homogeneizado com pipeta Pasteur. Uma gota de suspensão celular foi transferida para uma lâmina de vidro, onde foi feito o esfregaço. Após secagem das lâminas, estas foram fixadas em metanol absoluto durante $5 \mathrm{~min}$. e coradas em corante Giemsa por 15 minutos. Posteriormente, as lâminas foram lavadas em água corrente e secas em condições ambientais per noite. Para cada animal foram confeccionadas quatro lâminas, das quais duas foram utilizadas na contagem.

\section{Análise Citogenética}

Para a avaliação da atividade mutagênica do extrato, foram analisados micronúcleos de eritrócitos policromáticos em 2000 EPC, conforme Schmid (1975). Para a determinação da atividade citotóxica, foram computados 2000 EPC e determinada simultaneamente a freqüência de ENC. A confecção das lâminas e a contagem foram realizadas pelo procedimento duplo-cego e as lâminas foram examinadas no microscópio Olympus BH-2 em objetivo de imersão (10x100), utilizando-se duas lâminas para cada animal.

\section{AnÁlise Estatística}

$\mathrm{Na}$ análise da atividade mutagênica da planta, os resultados da freqüência da EPCMN induzidos com diferentes doses do EGb 761 foram comparados com o do grupo controle negativo (água destilada) e o do grupo controle positivo pelo t-Student, tendo sido considerados significativos valores de $\mathrm{P}<0,05$. $\mathrm{Na}$ avaliação da citotoxicidade da planta, a relação EPC/ENC com diferentes doses do extrato foi comparada com a do grupo controle negativo e a do grupo controle positivo utilizando-se o teste Qui-quadrado, tendo sido considerados significativos os valores de $\mathrm{P}<0,01$.

\section{Resultados}

Os resultados das freqüências de EPCMN e da relação EPC/ENC estão apresentados na Tabela 1 . Pode-se verificar que os resultados obtidos com o tratamento usando EGb 761 na dose de 50 $\mathrm{mg} / \mathrm{kg}$ do peso corpóreo do animal apresentaram média de 7,6 EPCMC/2000. Observou-se que com essa dose, a quantidade de EPCMN obtida foi similar à encontrada no controle negativo $(6,6$ EPCMN/2000) $(\mathrm{P}>0,05)$. Do mesmo modo, nas doses de $100 \mathrm{mg} / \mathrm{kg}$ e $200 \mathrm{mg} / \mathrm{kg}$, foram obtidas, respectivamente, médias de 6,8 EPCMN e 6,8 EPCMN. Esses resultados também não mostraram diferença significativa $(\mathrm{P}>0,05)$ em relação ao controle negativo $(\bar{X}=6,6)$. Assim, pode-se sugerir que o EGb 761 não apresentou ação genotóxica nas doses utilizadas. Todas as doses utilizadas de EGb 761 (50 mg/kg, $100 \mathrm{mg} / \mathrm{kg}$ e $200 \mathrm{mg} / \mathrm{kg}$ ) apresentaram diferença significativa em relação ao grupo controle positivo $\mathrm{MM}(4 \mathrm{mg} /$ $\mathrm{kg})(\mathrm{P}<0,05)$.

Em relação à citotoxicidade (Tabela 1 ), para todas as doses utilizadas $(50 \mathrm{mg} / \mathrm{kg}, 100$ $\mathrm{mg} / \mathrm{kg}$ e $200 \mathrm{mg} / \mathrm{kg}$ ), não foi constatada diferença significativa da relação EPC/ENC em relação ao grupo controle negativo $(\mathrm{P}>0,01)$. Todas as doses utilizadas apresentaram diferença significativa da relação EPC/ENC em relação ao grupo controle positivo $(\mathrm{P}<0,01)$. Assim, pode-se sugerir que o EGb 761 não exibiu atividade tóxica.

\section{Discussão}

O consumo de produtos naturais, incluindo extratos de plantas medicinais, tem aumentado acentuadamente nas últimas décadas. Apesar da ampla utilização desses medicamentos naturais, pouca informação encontra-se disponível sobre 
os riscos que oferecem à saúde humana. Sabese que as plantas não são tão "inócuas" como parecem, pois, durante o processo evolutivo, as plantas desenvolveram mecanismos naturais de defesa contra ataques de microrganismos, fungos, insetos e outros animais predadores (Pereira, 1999). No presente trabalho foi avaliada a atividade mutagênica e citotóxica do EGb 761 pelo teste do micronúcleo em eritrócitos policromáticos da medula óssea de ca-mundongos. $\mathrm{O}$ referido teste é um ensaio de curta duração que tem sido amplamente empregado para avaliar o potencial mutagênico (clastogênico e/ou aneugênico) e citotóxico de substâncias in vivo. Esse ensaio tem sido amplamente utilizado por ser de execução rápida, de grande sensibilidade e por utilizar animais vivos, o que inclui fatores como absorção, distribuiç̧ão e metabolismo da substância a ser avaliada (Sei-Ichi \& Isao, 2001). Como os camundongos apresentam padrões metabólicos similares aos humanos (Boone et al., 1990), a via de administração escolhida para a substância teste foi oral (v.o.). O tempo de tratamento escolhido foi de 24 horas, pois, segundo Heddle (1973), as células da medula óssea de mamífero levam de 22 a 24 horas para completar um ciclo de divisão.

Os resultados do presente estudo mostraram, conforme o esperado, que a freqüência de eritrócitos policromáticos micronucleados (EPCMN) foi elevada nos animais tratados com MMC (controle positivo) e baixa nos tratamentos com água destilada estéril (controle negativo). O EGb 761, nas doses de $50 \mathrm{mg} / \mathrm{kg}$, $100 \mathrm{mg} / \mathrm{kg}$ e $200 \mathrm{mg} / \mathrm{kg}$, não provocou aumento significativo ( $p>0,05)$ na freqüência de EPCMN em relação ao grupo controle negativo. Dessa maneira, não se observou a presença de ação genotóxica do extrato nas condições experimentais executadas. Todas as doses utilizadas do EGb 761 (50 mg/kg, $100 \mathrm{mg} / \mathrm{kg}$ e $200 \mathrm{mg} / \mathrm{kg}$ ) apresentaram diferença significativa em relação ao grupo controle positivo MMC $(4 \mathrm{mg} / \mathrm{kg})(\mathrm{P}<0,05)$. Esses resultados estão de acordo com o esperado.

Sabe-se que a relação entre EPC e ENC pode indicar a toxicidade de uma substância em ensaio. Segundo Mavournin et al. (1990), a razão entre a freqüência de EPC e ENC decresce quando a substituição dos EPC originados dos eritroblastos é deprimida. Pelos resultados obtidos, verificou-se que não houve decréscimo estatisticamente significativo da relação EPC/ENC em comparação ao controle negativo em todas as doses $(\mathrm{P}>0,05)$. Assim, foi observado que o EGb 761 não apresentou atividade citotóxica nas condições experimentais utilizadas.

\section{Conclusão}

Pelos resultados obtidos, pode-se concluir que o extrato de folhas de G. biloba. (EGb 761) não apresentou atividade mutagênica e citotóxica para todas as doses testadas nas condições

Tabela 1. Freqüência de micronúcleo (MN) em eritrócitos policromáticos (EPC) da medula óssea de camundongos e a relação EPC/ENC após 24 horas de tratamento com extrato de Ginkgo biloba (EGb761) em diferentes doses.

\begin{tabular}{|c|c|c|c|}
\hline \multirow{2}{*}{ Tratamento } & \multicolumn{2}{|c|}{ Eritrócitos policromáticos micronucleados (EPC- } & EPC/ENC \\
\cline { 2 - 4 } & Dados Individuais & $\overline{\mathrm{x}} \pm \mathrm{S}$ & $\overline{\mathrm{x}} \pm \mathrm{S}$ \\
\hline $50 \mathrm{mg} / \mathrm{kg}$ EGB p.o & $9,9,5,8,7$ & $7,6 \pm 1,67 \mathrm{ab}$ & $1,120 \pm 0,073 \mathrm{~cd}$ \\
\hline $100 \mathrm{mg} / \mathrm{kg}$ EGB p.o. & $8,6,8,8,4$ & $6,8 \pm 1,78 \mathrm{ab}$ & $0,971 \pm 0,15 \mathrm{~cd}$ \\
\hline $200 \mathrm{mg} / \mathrm{kg}$ EGB p.o. & $3,9,7,8,7$ & $6,8 \pm 2,28 \mathrm{ab}$ & $0,987 \pm 0,254 \mathrm{~cd}$ \\
\hline $\mathrm{C}(+):$ MMC 4 mg/kg i.p. & $30,36,31,42,33$ & $34,4 \pm 4,82 \mathrm{~b}$ & $0,483 \pm 0,110 \mathrm{~d}$ \\
\hline $\mathrm{C}(-): \mathrm{H}_{2} \mathrm{O}$ destilada & $8,5,9,4,7$ & $6,6 \pm 2,07 \mathrm{a}$ & $0,987 \pm 0,254 \mathrm{c}$ \\
\hline
\end{tabular}

a- Não foi constatada diferença significativa em relação ao grupo controle negativo pelo teste t-Student $(P>0,05)$;

b- Difere estatisticamente em relação ao grupo controle positivo (MMC) pelo teste t-Student considerando significativos valores de $\mathrm{P}<0,05$;

c- Não foi constatada diferença significativa em relação ao grupo controle negativo pelo teste Quiquadrado $(P>0,01)$;

d- Foi constatada diferença significativa em relação ao grupo controle positivo pelo teste Qui-quadrado $(P<0,01)$. 
experimentais utilizadas.

\section{REFERÊNCIAS}

Ahlemeyer, B., D. Selke, C. Schaper, S. Klumpp \& J. Krieglstein. 2001. Ginkgolic acids induce neuronal death and activate protein phosphatase type-2C. Eur. J. Pharmacol. 430: 1-7.

Amri, H., S.O. Ogwuegbu, N. Boujrad, K. Drieu \& V. Papadoppoulos. 1996. In vivo regulation of the peripheral-type benzodiazepine receptor and glucocorticoid synthesis by the Ginkgo biloba extract EGb 761 and isolated ginkgolides Endocrinology 137: 5707-5718.

Bastianetto, S. \& R. Quirion.2002. EGb 761 is a neuroprotective agent against beta-amyloid toxicity. Cell. Mol. Biol. 48: 693-697.

Boone, C.W., G.J. Kelloff \& W.E. Malone. 1990. Identification of candidate cancer chemopreventive agents and their evaluation in animal models and human clinical trials: a review. Cancer Res. 50: 2-9.

Chen, B., J. Cai, S.S. Song, X. Wang \& Z. Chen. 2005. Effects of Ginkgo biloba extract on cation currents in rat ventricular myocytes. Life Sciences 76: 1111-1121.

Choy, W.N. 2001. Regulatory Genetic toxicology tests. In: Choy, W. N. (ed.), Genetic Toxicology and Cancer Risk Assessment, p. 93-113, Marcel Dekker, Inc, New York.

Cohen-Salmon, C., P. Venault, B. Martin, M.J. Raffalli-Sébille, M. Barkats, F. Clostre, M.C. Pardon, Y. Christen, \& G. Chapouthier. 1997. Effects of Ginkgo biloba extract (EGb-761) on learning and possible actions on aging. J. Physiol. 91: 291-300.

Cordell, G.A. 1995. Changing strategies in natural products chemistry. Phytochemistry 40 : 1585-1612.

Couzinier, J.P. \& S. Mamatas. 1986. Basic and applied research in the pharmaceutical industry into natural substances, p. 57-61. In: D. Barton, Ollis, W.D. (Eds), Advances in Medicinal Phytochemistry. John Libbey Montrouge, Eurotext.

De Feudis, F.V. 2003. A brief history of EGb 761 and its therapeutic uses. Pharmacopsychiatry. 36: 52-57.

De Feudis, F.V. 1998. Ginkgo biloba extract (EGb 761) from chemistry to the clinic. Wies- baden, Ulstein Medical.

De Smet, P.A. 1995. Should herbal medicine-like products be licensed as medicines? Brit. Med. J. 310: 1023-1024.

Gaudineau, C.R. Beckerman \& S. Welbourn, Auclair, K. 2004. Inhibition of human P450 enzymes by multiple constituents of the Ginkgo biloba extract. Bioch. Bioph. Res. Comm. 318: 1072-1078.

Elovic, E.P. \& R.D. Zafonte. 2001. Ginkgo biloba: applications in traumatic brain injury. J. Head Trauma Rehabil. 16: 603-607.

Heddle, J.A. 1973. A rapid in vitro test for chromosomal damage. Mutat. Res. 18: 187-190.

Jaggy, H. \& E. Koch. 1997. The chemistry and biology of alkyphenols from Ginkgo biloba L. Pharmazie 10: 735-738.

Kleijnen, J. \& P. Knipschild. 1992. Ginkgo biloba for cerebral insufficiency. Br. J. Clin. Pharmac. 34: 352-358.

Koch, E., H. Jaggy, S.S Chatterjee. 2000. Evidence for immunotoxic effects of crude Ginkgo biloba L. leaf extracts using poplital lymph node assay in the mouse. Int. J. Immunopharmacol. 22: 229-236.

Kunitomo, M., K. Shinozuka, K. Umegaki, Y. Kubota, N. Tanaka, H. Mizuno, J. Yamauchi, K. Nakamura \& M. Kunitomo. 2002. Feeding of Ginkgo biloba extract (GBE) enhances gene expression of hepatic cytochrome P-450 and attenuates the hypotensive effect of nicardipine in rats. Life Sciences 70: 2783-2792.

Oken, B.S., D.M. Storzbach \& J.A. Kaye. 1998. The efficacy of Ginkgo biloba on cognitive function on Alzheimer's disease. Arch. Neurol. 55: 1409-1415.

Pereira, D.G. 1999. Avaliação do potencial mutagênico e/ou recombinogênico de Hyptidendron canun (pohl ex Benth) R. Harley em células germinativas e somáticas de Drosophila melanogaster. Dissertação de Mestrado, Universidade Federal de Goiás, Goiânia.

Porsolt, R.D., P. Martin, A. Lenegre, S. Fromage \& K. Drieu. 1990. Effects of an extract of Ginkgo biloba on "learned helplessness" and other models of stress in rodents. Pharmacol. Biochem. Behav. 36: 963-971.

Rapin, J.R., I. Lamproglou, K. Drieu \& F.V. De Feudis. 1994. Demonstration of the "anti- 
stress" activity of an extract of Ginkgo biloba using a discrimination learning task. Gen. Pharmacol. 25: 1009-1016.

Rioufol, G., S. Pietri, M. Culcasi, J. Loufoua, P. Staat, C. Pop, K. Drieu \& M. Ovise. 2003. Ginkgo biloba extract EGb 761 attenuates myocardial stunning in the pig heart. Basic Res. Cardiol. 98: 59-68.

Saller, R., J. Reichling \& O. Kristof. 1998. Phytotherapie - Behandlung ohne Ne-benwirkungen? Dtsch. Med. Wschr. 123: 58-62.

Schmid,W. 1975. The micronucleus test. Mutat. Res. 31: 9-15.

Sei-ichi, S. \& T. Isao. 2001. Short-term scre-ening method of the prediction for the prediction of carcinogenicity of chemical substances: current status and problems of an in vivo rodent micronucleus assay. J. Health Sci. 47: 1-8.

Shaw, D., C. Leon, S. Kolev \& V. Murray. 1997. Traditional remedies and food supplements. A 5-year toxicological study (1991-1995).
Drug Safety 17: 342-356.

Siegers, C.P. 1999. Cytotoxicity of alkylphenols from Ginkgo biloba. Phytomedicine 6: 281283.

Thiagaraja, G., S. Chandani, S.H. Rao, A.M. Samuni, K. Chandrasekaran \& D. Bala-subramanian. 2002. Molecular and cellular assessment of Ginkgo biloba extract as a possible ophthalmic drug. Exp. Eye Res. 75: 421-430.

Wadsworth, T.L. \& D.R. Koop. 2001. Effects of Ginkgo biloba extract (EGb 761) and quercetin on lipopolysaccharide-induced release of nitric oxide. Chem.-Biol. Interactions 137: 43-58.

Westendorf, J. \& J. Regan. 2000. Genotoxic and tumor promoting activity of ginkgolic acids in primary rat hepatocytes. Phytomedicine 7 (Suppl. II): 104.

Zhao, H.W. \& X.Y. Li. 2002. Ginkgolide A, B, and huperzine $\mathrm{A}$ inhibit nitric oxide-induced neurotoxicity. Int. Immunophar-macol. 2: 1551-1556.

Recebido em 24.III.2006 Aceito em 30.XI.2006 